\title{
Frequency addressing of nano-objects by electrical tuning of optical antennas
}

\author{
Costantino De Angelis, ${ }^{1, *}$ Andrea Locatelli, ${ }^{1}$ Daniele Modotto, ${ }^{1}$ Stefano Boscolo,${ }^{2}$ Michele Midrio, ${ }^{2}$ and \\ Antonio-D. Capobianco ${ }^{3}$ \\ ${ }^{1}$ Dipartimento di Ingegneria dell'Informazione, Università degli Studi di Brescia, Brescia 25123, Italy \\ ${ }^{2}$ Dipartimento di Ingegneria Elettrica, Gestionale e Meccanica, Università degli Studi di Udine, Udine 33100, Italy \\ ${ }^{3}$ Dipartimento di Ingegneria dell'Informazione, Università degli Studi di Padova, Padova 35131, Italy \\ *Corresponding author: costantino.deangelis@ing.unibs.it
}

Received December 22, 2009; revised March 4, 2010; accepted March 6, 2010; posted March 9, 2010 (Doc. ID 121926); published April 26, 2010

\begin{abstract}
We first analyze the equivalent circuit parameters of linear wire optical nano-antennas in uniaxial anisotropic media. We then exploit the electro-optic response of a bipolar nematic liquid crystal to demonstrate tuning of an optical antenna using a low frequency external electric field as the control mechanism. (C) 2010 Optical Society of America
\end{abstract}

OCIS codes: $350.4238,050.6624,260.3910$.

\section{INTRODUCTION}

Recent years have witnessed an impressive amount of research on optical devices conceived to efficiently couple free-space propagating light with localized light emitters or receivers, for a wide variety of potential applications, including nonlinear optics, efficient solar cells, near-field optical microscopy, and spectroscopy [1-12]. Beyond confining light of a given frequency at fixed locations, imposed by the structure geometry, there is a need for dynamical control of such hot-spots, for instance, to achieve selective frequency addressing of different nearby nanoobjects $[13,14]$. The possibility of dynamically tuning the resonance frequency of optical antennas might thus open new frontiers in probing of matter by optical means. To reach our goal, in this paper we analyze a linear wire aerial immersed in a uniaxial anisotropic medium.

The behavior of surface plasmon resonances in uniaxial anisotropic media has been already discussed both theoretically and experimentally in the literature $[15,16]$; in this framework both individual and coupled spherical nano-particles have been considered $[17,18]$. From the application viewpoint, focusing our attention to rod nanoparticles, the modeling requires one to solve two different aspects of the physical problem: the influence of anisotropy in the resonant behavior of a single nano-rod and in the coupling mechanism between two adjacent nano-rods. In terms of antennas and equivalent circuits what is needed is to determine the self-impedance and effective length of a cylindrical wire and the load impedance describing the role played by a small gap in it [5]. With these results at hand, we will then be able to discuss the tuning mechanism controlled by the director orientation for a linear wire optical antenna immersed in a nematic liquid crystal (LC).

The system to be considered in this paper is a wire an- tenna parallel to the $z$ axis (see Fig. 1) inside a nematic LC cell, i.e., a thin layer of LC placed between two parallel glass plates and rubbed so that the molecules are parallel to the $x$ axis (the director orientation). The material then acts as a uniaxial crystal (described by two different values of the electric permittivity: $\epsilon_{\|}$along the LC director and $\epsilon_{\perp}$ normal to the LC director). If an electric field is applied in the $z$ direction (by a voltage $V$ across transparent conductive electrodes coated in the glass plate), the resultant electric field forces tend to tilt the molecules from their original alignment (along the $x$ axis) toward the alignment of the field, and when the electric field is large enough most molecules tilt except those adjacent to the glass surface. When the electric field is removed the molecules tilt back to their original orientation parallel to the plates. As a consequence, the external steady or low frequency electric field can be used to switch the device between two states: in the presence of the low frequency external field the LC directors are parallel to the linear wire; in the absence of the external control the LC directors are orthogonal to the linear wire.

As a particular but representative example, we consider the response of the system when excited by a plane wave of amplitude $E_{0}$ impinging along the $x$ axis with its electric field parallel to the $z$ axis (see Fig. 1). In the following, we will always assume that the antenna has a total length $L$ of $82 \mathrm{~nm}$ (including the gap region, $g=2 \mathrm{~nm}$ ) and a radius $a$ of $5 \mathrm{~nm}$; moreover the antenna is made of silver, a low-loss metal whose permittivity at the frequencies of interest is well described by a classic Drude model: $\epsilon_{\mathrm{Ag}}=\epsilon_{0}\left(\epsilon_{\infty}-f_{p}^{2} /(f(f+i \gamma))\right)$, with $\epsilon_{\infty}=5, f_{p}=2.175 \mathrm{PHz}$, and $\gamma$ $=4.35 \mathrm{THz}$ [5]. The anisotropic medium wherein the antenna is embedded is a nematic LC E7 and we will use the known values of ordinary $\left(n_{0}=1.49\right)$ and extraordinary $\left(n_{e}=1.75\right)$ refractive indices in the infrared range $[19,20]$. 


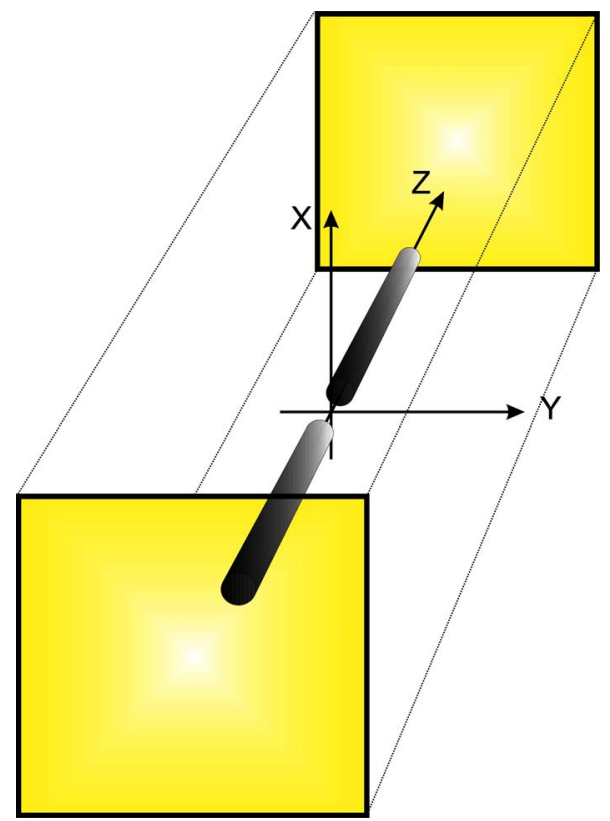

Fig. 1. (Color online) Schematic view of the cylindrical antenna (gray) inside the LC cell. The source (or the receiver) is intended to be in the gap of thickness $g=2 \mathrm{~nm}$; the director of the nematic LC can be oriented in the $(x, z)$ plane by controlling the applied voltage $V$.

\section{THÉVENIN EQUIVALENT CIRCUIT FOR A RECEIVING ANTENNA IN A UNIAXIAL ANISOTROPIC MEDIUM}

Antenna equivalent circuits are a key issue for designing nano-circuits connected to the nano-antennas by plasmonic waveguides. Indeed, it has recently been demonstrated that coupling between different plasmonic elements can be studied by using impedance matching techniques which are well established at the radio frequency $[5,6,14,21,22]$.

The Thévenin equivalent circuit of an antenna in the receiving mode is shown in Fig. 2, where $V_{\mathrm{oc}}$ is the opencircuit voltage, $Z_{A}$ is the antenna self-impedance, and $Z_{L}$ is the load impedance. Finite-element and time domain simulations [23,24] were both used and thoroughly cross checked to calculate the self-impedance $\left(Z_{A}\right)$ and the effective length $\left(l_{\text {eff }}\right)$ of the receiving antenna in order to gain access and make use of the equivalent circuit model schematically described in Fig. 2, where $V_{\text {oc }}=E_{0} l_{\text {eff. }}$ To determine the equivalent circuit parameters, instead of using a delta-gap source excitation $[25,26]$, we decided to exploit reciprocity and we numerically solved two different receiving situations: in both cases the receiving dipole is

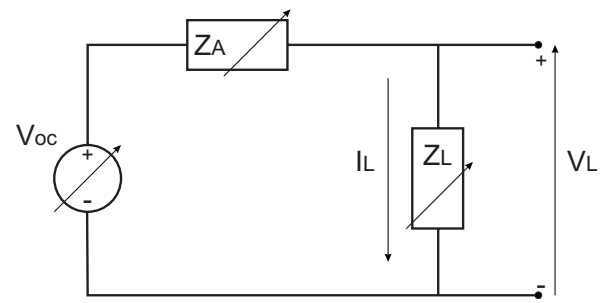

Fig. 2. Thévenin equivalent circuit in the receiving mode: $Z_{A}$, self-impedance of the antenna; $V_{\text {oc }}$, open-circuit voltage; $Z_{L}$, load impedance. excited by a plane wave impinging along the $x$ direction with the electric field parallel to the $z$ axis and we compute $V_{L}$ (voltage drop across the load) and $I_{L}$ (current through the load) as the line integral of the $z$ component of the electric field along the gap and the line integral of the magnetic field around the gap, respectively. In the first numerical experiment (case 1) the material in the gap is equal to the surrounding medium, while in the second situation (case 2) the gap is filled with a different medium. Note that this simple procedure permits one to avoid the use of lumped elements as excitation devices at the antenna terminals, thus allowing one to get a precise value of the antenna circuit parameters without perturbing the near-field region. Using subscripts 1 and 2 to refer to the two above described cases, we get

$$
\begin{gathered}
Z_{L 1,2}=\frac{V_{L 1,2}}{I_{L 1,2}}, \\
V_{L 1,2}=\frac{V_{\text {oc }} Z_{L 1,2}}{\left(Z_{A}+Z_{L 1,2}\right)},
\end{gathered}
$$

with $V_{\text {oc }}$ and $Z_{A}$ to be determined. Solving Eqs. (1) for the two unknown parameters we get
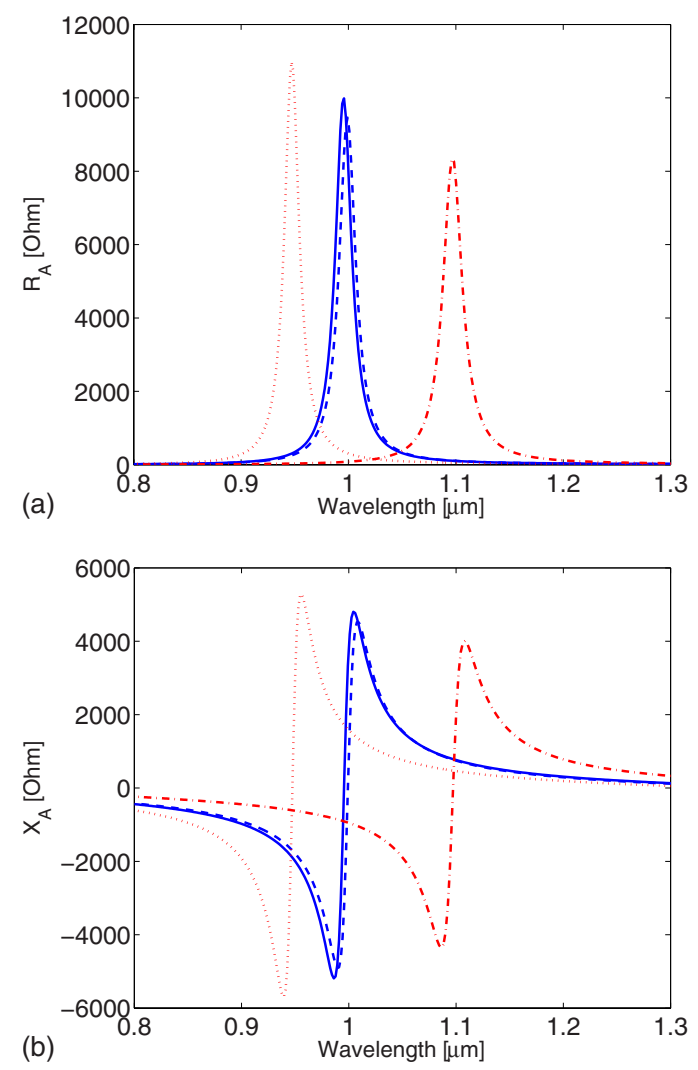

Fig. 3. (Color online) (a) $R_{A}=\Re e\left(Z_{A}\right)$, (b) $X_{A}=\Im m\left(Z_{A}\right)$ calculated from finite element method simulations: continuous (dashed) blue lines refer to the LC directors oriented orthogonal (parallel) to the wire antenna. The dashed-dotted red line refers to an isotropic medium with $n=n_{e}=1.75$, while the dotted red line refers to an isotropic medium with $n=n_{o}=1.49$. 


$$
\begin{gathered}
Z_{A}=Z_{L 1}\left(\frac{1-V_{L 1} / V_{L 2}}{V_{L 1} / V_{L 2}-Z_{L 1} / Z_{L 2}}\right)=\frac{V_{L 2}-V_{L 1}}{I_{L 1}-I_{L 2}}, \\
V_{\text {oc }}=V_{L 1} \frac{Z_{A}+Z_{L 1}}{Z_{L 1}} .
\end{gathered}
$$

In Fig. 3 we report the numerically evaluated antenna impedance between 225 and $375 \mathrm{THz}$, for the linear wire antenna immersed in the anisotropic medium with the LC directors oriented orthogonal (continuous line) and parallel (dashed line) to the wire antenna. Here and in the following we limit our attention to this frequency interval because it corresponds to the first open-circuit resonance, where strong field enhancement is expected. In Fig. 3(a) we report the real part, while in Fig. 3(b) we have the imaginary part of the self-impedance. Note that in the numerical simulations reported in this paper we need to tackle the full three-dimensional problem; in fact, due to the anisotropy of the surrounding medium, we cannot use the axial symmetry of the antenna structure to simplify the problem into an equivalent two-dimensional case, as usually done in isotropic media.

In Fig. 4 we report the numerically evaluated effective length $l_{\text {eff }}$ and the load impedance between 225 and 375 THz. In Fig. 4(a) we see the value of the effective length:

(a)
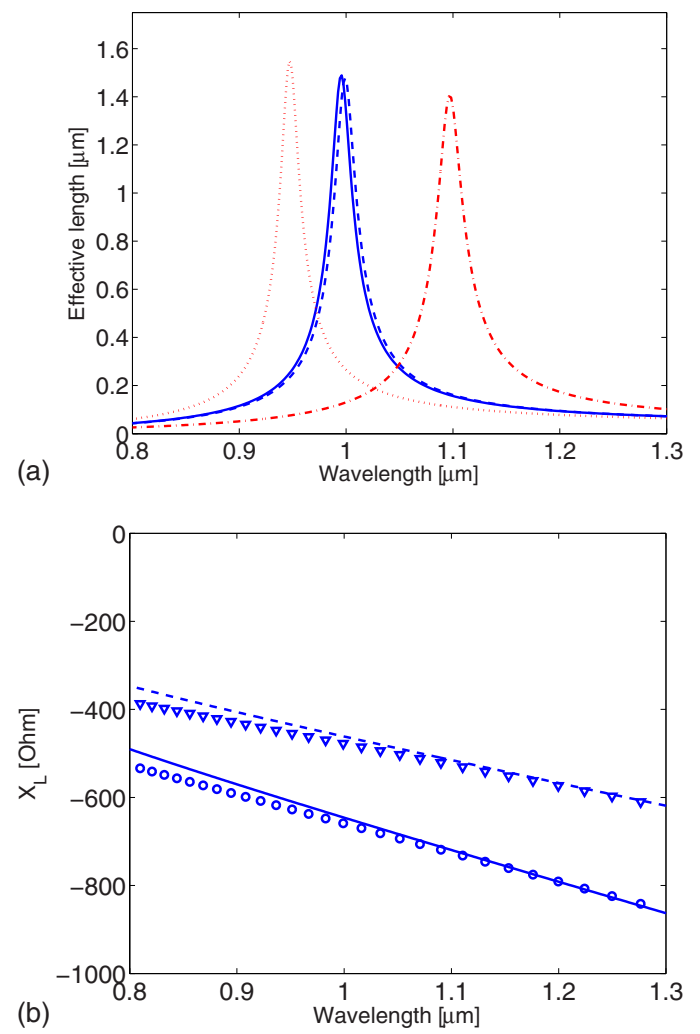

Fig. 4. (Color online) (a) Effective length: continuous (dashed) blue lines correspond to the LC director oriented orthogonal (parallel) to the wire antenna; the dashed-dotted red line refers to an isotropic medium with $n=n_{e}=1.75$, while the dotted red line refers to an isotropic medium with $n=n_{o}=1.49$; (b) load impedance: continuous (dashed) line corresponds to the results numerically obtained for the LC director oriented orthogonal (parallel) to the wire antenna; circles and triangles show the capacitive approximation of the gap region. continuous (dashed) line corresponds to the LC directors oriented orthogonal (parallel) to the wire antenna. In Fig. 4(b) we see the numerically computed value of the load impedance: continuous (dashed) line corresponds to the LC directors oriented orthogonal (parallel) to the wire antenna; in Fig. 4(b) we also report a load impedance estimation as the optical impedance of a nano-disk of height $g$, diameter $2 a$, and permittivity $\epsilon_{\perp, \|}$, when excited by an electric field of frequency $f$ parallel to its axis $[Z \propto$ $\left.-i g /\left(2 \pi^{2} f \epsilon a^{2}\right)\right][6]$ : circles (squares) correspond to the LC directors oriented orthogonal (parallel) to the wire antenna. Note that the capacitive nature of the load is a very reasonable approximation. As a useful comparison to enlighten the key features provided by the anisotropic environment, in Figs. 3(a), 3(b), and 4(a) we also show the circuit parameters $\left(Z_{A}\right.$ and $\left.l_{\text {eff }}\right)$ of the same antenna immersed in two different isotropic media: the low frequency dashed-dotted red line refers to an isotropic medium with $n=n_{e}=1.75$, while the high frequency dotted red line refers to an isotropic medium with $n=n_{o}=1.49$. Note that although we change the complete environment where the antenna is located, in the anisotropic case, as opposed to what is observed in the isotropic case, the effective length and self-impedance are almost unaffected by the orientation of the LC director; considerable changes in the circuit parameters affect mostly the load impedance $Z_{L}$ shown in Fig. 4(b) where we do not report the isotropic case since the high (low) index isotropic cases would be exactly superimposed to the two anisotropic cases with LC directors parallel (orthogonal) to the wire antenna. In agreement with the previous findings, we also note here that the principal wave of the infinite cylinder is only slightly influenced by the LC orientation, despite the effective index parameter of the infinite cylinder at $300 \mathrm{THz}$ goes from about 7.5 (when the surrounding medium is isotropic with $n=n_{o}=1.49$ ) to about 9.5 (when the host medium is isotropic with $n=n_{e}=1.75$ ).

\section{CIRCUIT MODELING AND ELECTRICAL TUNING OF FIELD ENHANCEMENT}

The possibility of achieving a strong field enhancement on a nanometer scale in the antenna feed gap is certainly one of the main reasons that triggered research in the area of optical antennas in the last years [4,7,8,27-29]. Here we will demonstrate that the unique properties offered by anisotropic media and in particular by nematic LCs can be used in this framework to electrically control the resonance frequency and hence as a tuning mechanism to address nano-objects at different frequencies. Let us consider the circuit model for the antenna in the receiving mode depicted in Fig. 2. $V_{\text {oc }}$ is the open-circuit voltage due to the incident field, and the voltage $V_{L}$ at the terminals of the gap capacitance is just what we need in order to calculate the electric field into the gap; in fact $\left|E_{z}(0,0,0)\right|$ $=V_{L} / g$. As a consequence the field enhancement reads $\left|E_{z}(0,0,0) / E_{0}\right|=V_{L} /\left(g E_{0}\right)$. The voltage $V_{L}$ has been calculated by writing the simple equation of the voltage divider $V_{L}=V_{\text {oc }} Z_{C} /\left(Z_{C}+Z_{A}\right)$ (Fig. 5 , circles), where $Z_{C}$ is the impedance associated with a pure capacitive model of the gap $[5,6,14]$. This result is compared with what one obtains through the full wave numerical solution of the 
problem modeling a plane wave (with an amplitude of $E_{0}=1 \mathrm{~V} / \mathrm{m}$ ) impinging on the antenna and recording the value of the electric field in the middle of the gap (Fig. 5, lines). In Fig. 5 dashed line and squares (continuous line and circles) refer to the case with the LC directors oriented parallel (orthogonal) to the wire antenna.

We observe that in principle the role played by the orientation of the directors of the nematic LC is twofold, as clearly enlightened by the equivalent circuit model. From one side the angle $\theta$ between the axis of the antenna and the anisotropy axis modifies the self-impedance and effective length of the antenna [Figs. 3(a), 3(b), and 4(a)]; from the other side, the permittivity of the medium along the $z$ axis changes the effective value of the load [Fig. 4(b)]. The two mechanisms together are responsible for the tuning of the resonance of the antenna; however, looking at the results reported in Figs. 3-5, we can now clearly state, contrary to what one might expect, that the change in the load (i.e., the change in the coupling between the two antenna sides) is playing here the dominant contribution to the overall effect; thus we can tune a load at the nanoscale using a voltage applied on electrodes of a micrometer scale.

In order to summarize the possibilities offered by the external electric field control, we have also considered the case where the external field is continuously varied from zero (LC directors orthogonal to the antenna axis) to $V_{\max }=6 \mathrm{~V}$, the voltage necessary to assume almost complete orientation of the directors along the electric field lines. In the following we assume the following law for the orientation angle $\theta$ (the angle of the director with respect to the $x$ axis): $\theta=0$ for $V \leq V_{c}$ and $\theta=\pi / 2-2 \arctan \exp ($ $\left.-\left(V-V_{c}\right) / V_{0}\right)$ for $V>V_{c}$, with $V_{c}=1 \mathrm{~V}$ and $V_{0}=2 \mathrm{~V}$ [20]. We thus get the following expression of the permittivity matrix as a function of the orientation angle $\theta$ :

$$
\begin{gathered}
\epsilon=\left(\begin{array}{ccc}
\epsilon_{1,1} & 0 & \epsilon_{1,3} \\
0 & \epsilon_{\perp} & 0 \\
\epsilon_{3,1} & 0 & \epsilon_{3,3}
\end{array}\right), \\
\epsilon_{1,1}=\epsilon_{\|} \cos ^{2}(\theta)+\epsilon_{\perp} \sin ^{2}(\theta), \\
\epsilon_{3,3}=\epsilon_{\perp} \cos ^{2}(\theta)+\epsilon_{\|} \sin ^{2}(\theta), \\
\epsilon_{1,3}=\epsilon_{3,1}=\left(\epsilon_{\|}-\epsilon_{\perp}\right) \cos (\theta) \sin (\theta) .
\end{gathered}
$$

Using $\epsilon_{\|}=3.06$ and $\epsilon_{\perp}=2.22$, in Fig. 6 we report the value of the resonance frequency of the antenna as a function of the electric field applied across the electrodes. Note that the external electric field can in this case be used as a tuning mechanism to move the resonance frequency inside $\mathrm{a} \simeq 6.5 \mathrm{THz}$ frequency bandwidth.

In Fig. 6 we also report the value of the field enhancement at two given frequencies $(f=265$ and $271 \mathrm{THz})$ as a function of the applied voltage $V$ : the results clearly show that in both the cases the electric control can be used to switch the device from the on state (high enhancement value) to the off state (low enhancement value), and vice versa. Although the reported switching extinction ratio is not large, possible ways to overcome this limitation can be

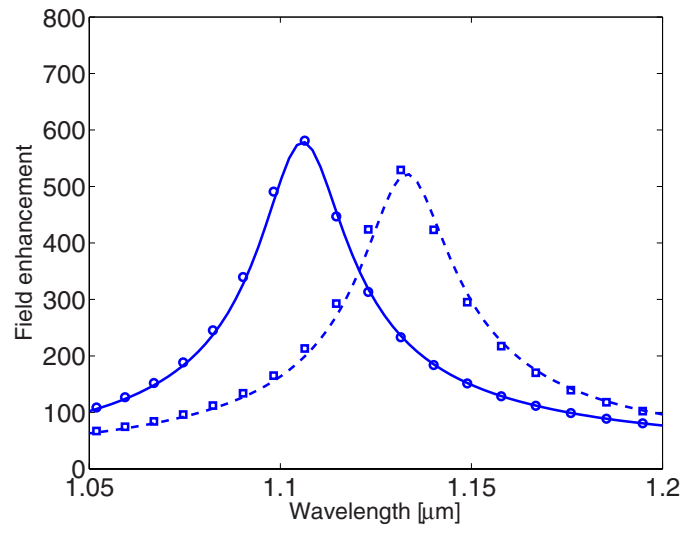

Fig. 5. (Color online) Field enhancement versus frequency: continuous (dashed) line refers to the case with the LC directors oriented orthogonal (parallel) to the wire antenna as computed from full numerical simulation of the problem at hand; circles and squares refer to the values as obtained from the equivalent circuit model.

sought, for example, exploiting higher birefringence or more frequency selective aerials.

\section{CONCLUSIONS}

In conclusion, we have analyzed the equivalent circuit of an optical dipole in a uniaxial anisotropic medium. We have studied the resonance frequency of the structure as a function of the relative orientation between the dipole
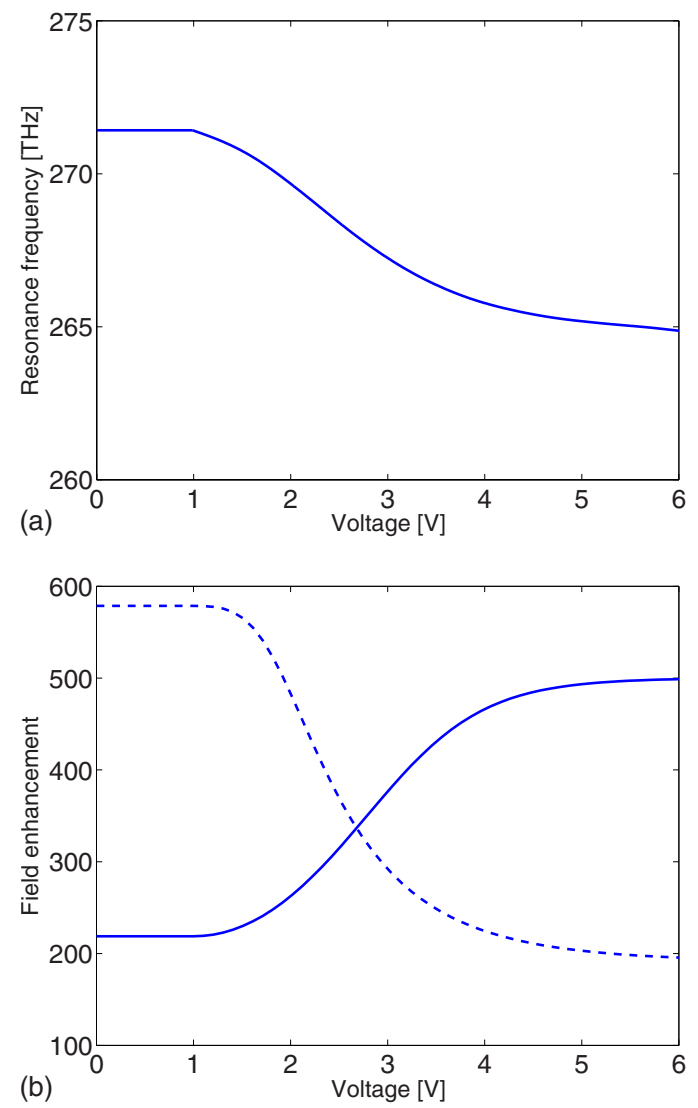

Fig. 6. (Color online) (a) Resonance frequency versus applied electric voltage $V$; (b) field enhancement versus applied voltage $V$ at $f=265 \mathrm{THz}$ (continuous line) and at $f=271 \mathrm{THz}$ (dashed line). 
and the index ellipsoid of the anisotropic medium. In this framework we have demonstrated that one can tune a load at the nanoscale by means of a voltage applied on electrodes of a micrometer scale. Using as a reference example a nematic liquid crystal (LC) we have described the possible use of these properties to tune the frequency resonance of a simple dipole antenna in order to address nano-objects at different frequencies.

\section{ACKNOWLEDGMENTS}

M. Midrio has carried out this work in the framework of an agreement for the selection of researchers of recognized prestige between Carlos III University of Madrid and Banco Santander.

\section{REFERENCES}

1. S. A. Maier, Plasmonics: Fundamentals and Applications (Springer, 2007).

2. E. Ozbay, "Plasmonics: merging photonics and electronics at nanoscale dimensions," Science 311, 189-193 (2006).

3. P. Bharadwaj, B. Deutsch, and L. Novotny, "Optical antennas," Adv. Opt. Photon. 1, 438-483 (2009).

4. P. Mühlschlegel, H. J. Eisler, O. J. F. Martin, B. Hecht, and D. W. Pohl, "Resonant optical antennas," Science 308, 1607-1609 (2005).

5. A. Alù, and N. Engheta, "Input impedance, nanocircuit loading, and radiation tuning of optical nanoantennas," Phys. Rev. Lett. 101, 043901 (2008).

6. A. Alù and N. Engheta, "Tuning the scattering response of optical nanoantennas with nanocircuit loads," Nat. Photonics 2, 307-310 (2008).

7. T. H. Taminiau, F. D. Stefani, F. B. Segerink, and N. F. Van Hulst, "Optical antennas direct single-molecule emission," Nat. Photonics 2, 234-237 (2008).

8. R. L. Olmon, P. M. Krenza, A. C. Jones, G. D. Boreman, and M. B. Raschke, "Near-field imaging of optical antenna modes in the mid infrared," Opt. Express 16, 20295-20305 (2008).

9. R. A. Davoyan, I. V. Shadrivov, and Y. S. Kivshar, "Quadratic phase matching in nonlinear plasmonic nanoscale waveguides," Opt. Express 17, 20063-20068 (2009).

10. D. Cialla, R. Siebert, U. Hubner, R. Moller, H. Schneidewind, R. Mattheis, J. Petschulat, A. Tunnermann, T. Pertsch, B. Dietzek, and J. Popp, "Ultrafast plasmon dynamics and evanescent field distribution of reproducible surface-enhanced Raman-scattering substrates," Anal. Bioanal. Chem. 394, 1811-1818 (2009).

11. J. Aizpurua, G. W. Bryant, L. J. Richter, and F. J. G. de Abajo, "Optical properties of coupled metallic nanorods for field-enhanced spectroscopy," Phys. Rev. B 71, 235420 (2005).

12. O. L. Muskens, V. Giannini, J. A. Sanchez-Gil, and J. G. Ri- vas, "Strong enhancement by the radiative decay rate of emitters by single plasmonic nanoantennas," Nano Lett. 7, 2871-2875 (2007).

13. G. Volpe, S. Cherukulappurath, R. J. Parramon, G. MolinaTerriza, and R. Quidant, "Controlling the optical near field of nanoantennas with spatial phase-shaped beams," Nano Lett. 9, 3608-3611 (2009).

14. A. Locatelli, C. De Angelis, D. Modotto, S. Boscolo, F. Sacchetto, M. Midrio, A. D. Capobianco, F. M. Pigozzo, and C. G. Someda, "Modeling of enhanced field confinement and scattering by optical wire antennas," Opt. Express 17, 16792-16800 (2009).

15. S. Y. Park and D. Stroud, "Surface-enhanced plasmon splitting in a liquid-crystal-coated gold nanoparticle," Phys. Rev. Lett. 94, 217401 (2005).

16. W. Dickson, G. A. Wurtz, P. R. Evans, R. J. Pollard, and A. V. Zayats, "Electronically controlled surface plasmon dispersion and optical transmission through metallic hole arrays using liquid crystal," Nano Lett. 8, 281-286 (2008).

17. J. Muller, C. Sonnichsen, H. von Poschinger, G. von Plessen, T. A. Klar, and J. Feldmann, "Electrically controlled light scattering with single metal nanoparticles," Appl. Phys. Lett. 81, 171-173 (2002).

18. J. Berthelot, A. Bouhelier, C. J. Huang, J. Margueritat, G. Colas-des-Francs, E. Finot, J. C. Weeber, A. Dereux, S. Kostcheev, H. I. El Ahrach, A. L. Baudrion, J. Plain, R. Bachelot, P. Royer, and G. P. Wiederrecht, "Tuning of an optical dimer nanoantenna by electrically controlling its load impedance," Nano Lett. 9, 3914-3921 (2009).

19. A. Fernandez-Nieves, D. R. Link, D. Rudhardt, and D. A. Weitz, "Electro-optics of bipolar liquid crystal droplets," Phys. Rev. Lett. 92, 105503 (2004).

20. T. S. Perova, V. A. Tolmachev, E. V. Astrova, Yu. A. Zharova, and S. M. O'Neill, "Tunable one-dimensional photonic crystal structures based on grooved Si infiltrated with liquid crystal E7," Phys. Status Solidi C 4, 1961-1965 (2007).

21. J. Wen, S. Romanov, and U. Peschel, "Excitation of plasmonic gap waveguides by nanoantennas," Opt. Express 17, 5925-5932 (2009).

22. J. S. Huang, T. Feichtner, P. Biagioni, and B. Hecht, "Impedance matching and emission properties of nanoantennas in an optical nanocircuit," Nano Lett. 9, 1897-1902 (2009).

23. COMSOL Multiphysics, 3.5, COMSOL Inc., http:// www.comsol.com/.

24. CST Microwave Studio 2009, Darmstadt, Germany.

25. C. A. Balanis, Antenna Theory, 3rd ed. (Wiley, 2005).

26. S. J. Orfanidis, Electromagnetic Waves and Antennas (2008); available online at http://www.ece.rutgers.edu/ orfanidi/ewa/.

27. J. J. Greffet, "Nanoantennas for light emission," Science 308, 1561-1563 (2005).

28. L. Novotny, "Optical antennas tuned to pitch," Nature $\mathbf{4 5 5}$, 887 (2008).

29. P. Ghenuche, S. Cherukulappurath, T. H. Taminiau, N. F van Hulst, and R. Quidant, "Spectroscopic mode mapping of resonant plasmon nanoantennas," Phys. Rev. Lett. 101, 116805 (2008). 